\title{
Automated Quantification of Tumor Viability in a Rabbit Liver Tumor Model after Chemoembolization Using Infrared Imaging
}

Hadrien D'inca, ${ }^{*}$ Julien Namur, ${ }^{\dagger}$ Saida Homayra Ghegediban, ${ }^{\dagger \dagger}$ Michel Wassef, ${ }^{\dagger \dagger}$ Florentina Pascale, ${ }^{\S}$ Alexandre Laurent, ${ }^{\S \Phi \|}$ and Michel Manfait*

\begin{abstract}
From the Median Unit,* University of Reims Champagne-Ardenne, Centre National de la Recherche Scientifique, Matrice Extracellulaire et Dynamique Cellulaire, Reims, the ArchimMed SARL, ${ }^{\dagger}$ Jouy en Josas; the Department of Pathology, ${ }^{\ddagger}$ Lariboisiere Hospital, Assistance Publique-Hôpitaux de Paris, Paris; the Research Center for Interventional Imagery, ${ }^{\S}$ Assistance Publique-Hôpitaux de Paris and National Institute for Agricultural Research, Jouy-En-Josas; the Department of Neuroradiology, "Hôpital Lariboisère, Assistance Publique-Hôpitaux de Paris, Paris; and the Materials and Complex Systems Laboratory," Centre National de la Recherche Scientifique, Paris, France
\end{abstract}

\author{
Accepted for publication \\ March 16, 2015. \\ Address correspondence to \\ Hadrien D'inca, Ph.D., Unité \\ Median, University of Reims \\ Champagne-Ardenne, Centre \\ National de la Recherche Sci- \\ entifique, Matrice Extracellu- \\ laire et Dynamique Cellulaire, \\ 51 rue Cognacq Jay, 51095 \\ Reims Cedex, France. E-mail: \\ hadrien.dinca@etudiant.univ- \\ reims.fr or dinca.hadrien@ \\ hotmail.fr.
}

\begin{abstract}
The rabbit VX2 tumor is a fast-growing carcinoma model commonly used to study new therapeutic devices, such as catheter-based therapies for patients with inoperable hepatocellular carcinoma. The evaluation of tumor viability after such locoregional therapies is essential to directing hepatocellular carcinoma management. We used infrared microspectroscopy for the automatic characterization and quantification of the VX2 liver tumor viability after drug-eluting beads transarterial chemoembolization (DEB-TACE). The protocol consisted of K-means clustering followed by principal component analysis (PCA) and linear discriminant analysis (LDA). The K-means clustering was used to classify the spectra from the infrared images of control or treated tumors and to build a database of many tissue spectra. On the basis of this reference library, the PCALDA analysis was used to build a predictive model to identify and quantify automatically tumor viability on unknown tissue sections. For the DEB group, the LDA model determined that the surface of tumor necrosis represented $91.6 \% \pm 8.9 \%$ (control group: $33.1 \% \pm 19.6 \%$; Mann-Whitney $P=0.0004$ ) and the viable tumor $2.6 \% \pm 4 \%$ (control group: $62.2 \% \pm 15.2 \%$; Mann-Whitney $P=0.0004)$. Tissue quantification measurements correlated well with tumor necrosis $(r=0.827, P<0.0001)$ and viable tumor $(r=0.840, P<0.0001)$. Infrared imaging and PCA-LDA analysis could be helpful for easily assessing tumor viability. (Am J Pathol 2015, 185: 1877-1888; http://dx.doi.org/10.1016/j.ajpath.2015.03.023)
\end{abstract}

The VX2 tumor model originates from a squamous cell carcinoma that developed as a result of malignant changes in the cells of a Shope virus-induced skin papilloma in a domestic rabbit. $^{1,2}$ This tumor model is serially transplantable in allogenic adult rabbits, easily implantable, and grows quickly in many types of organs, such as lungs, ${ }^{3}$ liver, ${ }^{4}$ or rectum. Therefore, the VX2 tumor blood supply is almost entirely from the hepatic artery, similar to that of humans, ${ }^{6}$ and rabbit hepatic arteries are large enough to permit hepatic artery catheterization. ${ }^{7}$ This makes the VX2 tumor a common animal model for the preclinical evaluation of new anticancer treatments $^{8-11}$ and for new therapeutic devices, such as catheter-based therapies for patients with inoperable hepatocellular carcinoma.
The evaluation of tumor response after locoregional therapies is essential in directing management for hepatocellular carcinoma. An understanding of the various therapeutic strategies and their posttherapy imaging appearance is essential for accurately assessing treatment response. The evaluation of tumor response should include not only anatomical evaluation, such as reduction in tumor size, but also the reduction of tumor viability, the degree of induced

\footnotetext{
Supported by ArchimMed SARL.

Disclosures: J.N., S.H.G., and F.P. are employed by ArchimMed SARL, and M.M. receives consulting fees or other remuneration from ArchimMed SARL.

Portions of this work were presented at the 15th European Conference on the Spectroscopy of Biological Molecules, August 25-30, 2013, Oxford, UK, and published in the proceedings.
} 
necrosis, ${ }^{12-14}$ and the biochemical changes that occur inside tumor tissues.

During the past decade, there has been considerable interest in spectroscopy-based microscopies, which use the intrinsic optical properties of the tissues to produce histologic images without staining or exogenous markers. It was first revealed in the 1950s that neoplastic and normal tissues could be discriminated based on their infrared absorption spectra $^{15,16}$ due to the differences in their biochemical composition. Infrared spectra directly provide a multivariate nonperturbing molecular description able to distinguish and quantify histologic constituents from tissues. Chemical concentrations are quantified by spectral absorbance properties at specific frequencies, and subtle molecular structural changes are indicated by spectral peak shifts, band shapes, and relative intensity changes. ${ }^{17,18}$ The coupling of the infrared spectrometer with an imaging system (microspectroscopy) further permitted to combine the measured spectrum to a spatial position on the sample and to record infrared images directly on thin tissue sections. Comprehensive studies using this technique revealed the potential for fast, stain-free, nondestructive molecular histopathologic analysis with a high spatial resolution and established the capability of infrared microspectroscopy (IRMS) to complement histopathologic tools for cancerous tissue diagnosis in different organs. ${ }^{19-25}$ The most recent developments in IRMS aimed at automating the procedure of tissue recognition and quantification by using statistical methods and prediction algorithms. ${ }^{26-31}$ These methods are based on the measurement of a large number of tissue specimens. The information gathered from this large data set is used to produce a reference spectral library of each tissue type. On the basis of this spectral library, a predictive model is elaborated and validated. Applying a model to a new tissue section generates a false colored image in which each color corresponds to a type of tissue and where the surface of each tissue is automatically calculated. Once the model is set up and validated, it can be applied to a new infrared image in only 1 minute. The infrared imaging technique is a solution to visualize on the same image the morphologic information and the molecular composition of tissues. It appears to be a helpful technique for studying objectively and quantitatively tumor response.

Our aim was to validate the use of IRMS to automate the recognition and the quantification of VX2 liver tumor viability after treatment with drug-eluting beads transarterial chemoembolization (DEB-TACE). We worked on a pool of untreated VX2 tumors and a pool of DEB-TACE-treated tumors. First, the infrared spectra characteristics of each tissue of interest were recorded and a prediction model of tissue types was developed. Second, the model was applied to a set of new test VX2 samples to assess the surface of viable and necrotic tumor. A validation procedure was included at each step of the data processing. Infrared results were correlated by histopathologic analysis as standard of reference.

\section{Materials and Methods}

\section{Animal Model and Tissue Samples}

This study was approved by the Animal Care Committee and was performed in accordance with our institutional guidelines. Adult New Zealand white rabbits weighing 6 to $8 \mathrm{lb}$ underwent implantation of rabbit VX2 tumor in the liver. The tumors were induced by an injection of a VX2 cells suspension $\left(0.25 \times 10^{6}\right.$ cells $/ \mathrm{mL}$ ) directly in the liver. We included 27 rabbits with VX2 liver tumors: 16 rabbits were subjected to a DEB-TACE treatment and compared with a control group of 11 rabbits. Animals from DEB-TACE group were treated after 12 days of tumor development and were euthanized 3 days after the embolization procedure (15 days of tumor development). In the same model, one experiment on our laboratory found a significant increase of tumor necrosis 3 days after embolization compared with untreated tumors. Animals in the control group were euthanized after 14 days of tumor development. Then, tumor-bearing livers were resected and samples were formalin fixed and paraffin embedded. Two adjacent sections were cut from each sample using a microtome. The first section (10- $\mu \mathrm{m}$ thick) was mounted on a calcium fluoride window suitable for IRMS. The second section $(5-\mu \mathrm{m}$ thick) was put on a standard glass slide, dewaxed, and rehydrated by means of successive baths of xylene and alcohol and stained with hematoxylin-eosinsaffron (HES) to serve as a control for infrared imaging.

\section{Fourier Transform IRMS}

Infrared spectral images were collected with an infrared microscope (Spectrum Spotlight 300 Imaging System, PerkinElmer, Courtaboeuf, France) coupled to a Spectrum One Fourier transform infrared (FTIR) spectrometer using the image mode. The device is equipped with a nitrogen-cooled mercury cadmium telluride 16-pixel-line detector for imaging and a computer-controlled stage to collect large spectroscopic images from a sample. The microscope was isolated in a venting Plexiglas housing to enable purging with dry air and to eliminate atmospheric interferences. Before acquisition, a visible image of the sample was recorded and the area of interest was selected by comparison to the corresponding H\&E-stained adjacent section.

In this study, 38 spectroscopic images were recorded from VX2 liver tumors and could vary in size from $8 \times 8 \mathrm{~mm}\left(64 \mathrm{~mm}^{2}\right)$ to $10 \times 10 \mathrm{~mm}\left(\right.$ or $\left.100 \mathrm{~mm}^{2}\right)$. Each pixel sampled a $25 \times 25 \mu \mathrm{m}\left(625 \mu^{2}\right)$ area at the sample plane, providing images that contained 120,000 to 160,000 individual infrared spectra (depending on the size of the image). Spectral data were acquired in transmission mode. All spectral measurements were recorded using a spectral resolution of $4 \mathrm{~cm}^{-1}$ and two scans per pixels, each spectrum containing 1601 values of absorbance, spanning the spectral range of $800 \mathrm{~cm}^{-1}$ to $4000 \mathrm{~cm}^{-1}$. A background spectrum was collected (75 accumulations, $4-\mathrm{cm}^{-1}$ resolution) outside the sample (on the calcium fluoride window 
Table 1 Sample Repartition in Each Study Group

\begin{tabular}{lllllll}
\hline & \multicolumn{2}{l}{ KM followed by PCA-LDA } & & \multicolumn{2}{l}{ Test samples } \\
\cline { 2 - 6 } Sample & CTRL & DEB-TACE & & CTRL & DEB-TACE & Total \\
\hline Tumors & 4 & 3 & 7 & 13 & 27 \\
Tissue sections and infrared images & 7 & 7 & 7 & 17 & 38 \\
\hline
\end{tabular}

CTRL, control; DEB-TACE, drug-eluting beads transarterial chemoembolization; KM, K-means; PCA-LDA, principal component analysis and linear discriminant analysis.

area) to ratio against the single-beam spectra. The resulting spectra were then automatically converted into absorbance.

\section{Infrared Images Preprocessing and Construction of a Predictive Model}

All subsequent data treatment protocols were performed with Spectrum image-Spotlight version 300 (PerkinElmer), Opus version 5.5 (Bruker Optik, Ettlingen, Germany), and Matlab version 7.12 (Mathworks, Natick, MA) software using protocols validated in our laboratory. All preprocessing and processing data were obtained directly from spectral images in the infrared absorption range of 900 to $1800 \mathrm{~cm}^{-1}$ (451 values of absorbance) considered as the most informative region.

Numerical Dewaxing and Poor-Quality Spectra Elimination Paraffin exhibits strong absorption bands at 1368 and 1467 $\mathrm{cm}^{-1}$. To correct the contribution of paraffin in FTIR spectra, we used an automated processing method based on extended multiplicative signal correction (EMSC) validated and commonly used in our laboratory. ${ }^{32,33}$ It is a numerical dewaxing without xylene treatment (chemical dewaxing). Briefly the EMSC algorithm constrain the bands of paraffin at the same intensity on all tissue spectra. The variations of the paraffin bands intensity are eliminated from tissue spectra. The EMSC method was also used for eliminating the infrared spectra with low signal-to-nose ratio. ${ }^{34}$

Consequently, the result of classification process [K-means $(\mathrm{KM})$ and linear discriminant analysis (LDA)] is based only on the spectral differences between each type of tissue. The spectra were paraffin and baseline corrected in the same step, and finally they were vector normalized on the 900- to $1800-$ $\mathrm{cm}^{-1}$ spectral range. For a more comprehensive description of the EMSC method, the reader should refer to previous articles. $^{33,35}$

\section{Construction of a Spectral Database by Unsupervised KM Classification}

On a first series of 14 infrared images (Table 1), we performed a KM classification. This statistical classification method gathered spectra that have similar spectral characteristics in clusters whose number is determined by the operator. ${ }^{24,29,32,36}$ Then, the pixel on infrared image (coordinates $\mathrm{x}$ and $\mathrm{y}$ ) corresponding to the spectrum is colored with the same color as the cluster. The color is randomly attributed to each cluster by the Matlab software. The result of the KM analysis is a false color image in which each color corresponds to a cluster. All the eliminated spectra by EMSC algorithm were colored as white pixels in the KM clustered images (spectra with low signal-to-nose ratio). Each image was analyzed independently; thus, colors of the different KM images were not comparable. The number of clusters varied from 2 to 10 . The accurate number of clusters was chosen with the pathologist in such a way that each tissue type identified on HES-stained sections was represented by at least one cluster. Each cluster had a unique histologic assignment.

To confirm the KM clustering, a total of 255 areas measuring $200 \mu \mathrm{m} \times 200 \mu \mathrm{m}$ each were selected on HES-stained tissue sections in zones of viable tumor, tumor necrosis, fibrosis, normal liver parenchyma, and liver parenchyma necrosis $(65,75,45,40$, and 30 areas, respectively). Each area was then located on the $14 \mathrm{KM}$ images to determine whether each cluster color corresponded to a particular tissue. Areas that contained multiple clusters were considered unallocated areas. We calculated the sensitivity of each cluster by dividing the number of areas correctly matched by the sum of true-positive and falsenegative results. Then, we calculated the specificity of each cluster by dividing the number of areas correctly matched by the sum of true-negative and false-positive results. The spectra of validated $\mathrm{KM}$ clusters were used to create a database containing thousands of spectra assigned to a specific type of tissue: viable tumor, tumor necrosis, fibrosis, liver parenchyma, and liver parenchyma necrosis.

\section{Construction of a Predictive Model by Combining PCA and LDA}

On the basis of the tissue spectral database, LDA looks for the variables containing both the greatest interclass variance and the smallest intraclass variance. Because we analyzed a huge quantity of spectra in the same time (approximately 430,000), we choose to apply a principal component analysis (PCA) before the LDA. The PCA is a commonly used spectral data processing method that reduces the size of the data while still retaining the variance. This variance is represented by principal components. The resulting scores were then used as inputs for LDA. The PCA-LDA model obtained is a linear combination of the variables to discriminate between the classes.

To evaluate the accuracy of the PCA-LDA model, the database was randomly divided into two uneven sets of spectra: a training set corresponding to two-thirds of spectra and a validation set corresponding to one-third of spectra. The training data set was used to establish and optimize the PCA and 

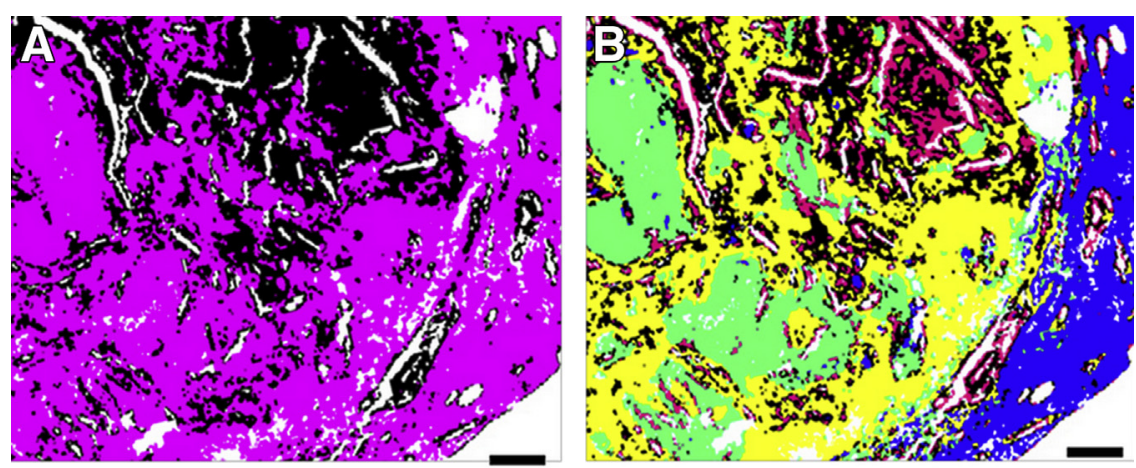

Figure $1 \quad V X 2$ histologic sections analyzed by infrared imaging and $\mathrm{K}$-means clustering $(\mathbf{A}-\mathbf{C})$ or stained with hematoxylin-eosin-saffron (D). A-C: Infrared images computed by K-means (KM) analysis in 2, 5, and 8 clusters, respectively. The color was arbitrary attributed to each cluster. All the eliminated spectra by extended multiplicative signal correction algorithm were colored as white pixels in the KM images. D: Tissue section stained with hematoxylin-eosin-saffron. The tissue section comprises a large region of viable tumor tissue (a), liver parenchyma (b), intratumoral fibrosis (c), and intratumoral necrosis (d). Scale bar $=1$ $\mathrm{mm}$.

LDA parameters that would provide the best possible classification. The validation data set, which is labeled with the correct answer, was used to test the accuracy of LDA model. The confrontation between the training set and the validation set gives the confusion matrix of the prediction model. The sensitivity of each class is calculated by dividing the number of spectra labeled as true-positive results by the sum of the number of true-positive and false-negative results. The specificity of each class is calculated by dividing the number of spectra labeled as true-negative results by the sum of true-negative and false-positive results. We chose the PCA-LDA model that displayed the best sensitivity and specificity for the five tissue types of interest.

\section{Application of the Predictive Model on Test Samples and Tissues Surface Quantification}

Once the model is validated, it can be applied to new test samples. ${ }^{26,29,30}$ In this study, the predictive model was applied to infrared images of 24 new test tumor sections (Table 1) from the 20 remaining tumors. Unidentified test spectra were analyzed by the PCA-LDA model that identified their tissue classes and colored them in accordance with their class color (dark blue color to represent tumor necrosis, yellow for viable tumor, blue for the fibrosis, green for liver parenchyma, and orange for liver parenchyma necrosis). When the maximum probability for a pixel was $<0.75$, the tissue assignment was considered ambiguous by the model and the pixel was colored in black. The result of the PCA-LDA model analysis is a false color image for which each color corresponds to a type of tissue. The LDA images obtained were quantitatively compared again to control HES-stained adjacent section as for KM images validation step. To confirm the LDA classification, a total of 300 areas measuring $200 \mu \mathrm{m} \times 200 \mu \mathrm{m}$ each were selected on HES-stained tissue sections in zones of viable tumor, tumor necrosis, fibrosis, normal liver parenchyma, and liver parenchyma necrosis $(66,77,53,56$, and 48 areas, respectively).

The number of pixels corresponding to each tissue on LDA images was automatically recorded by the algorithm and used to calculate the percentage of tumor surface occupied by viable tumor or necrotized areas. The values obtained on LDA images were compared with histologic measurements previously obtained on digitalized stained sections from the same paraffinized samples (NanoZoomer 2.0 HT slide scanner at a $\times 20$ objective, Hamamatsu,

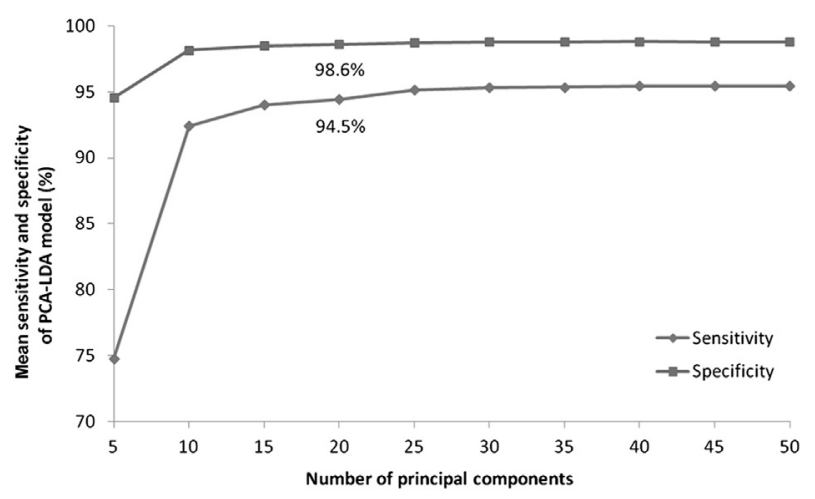

Figure 2 Mean sensitivity and specificity of the principal component analysis and linear discriminant analysis model according to the number of principal components. 

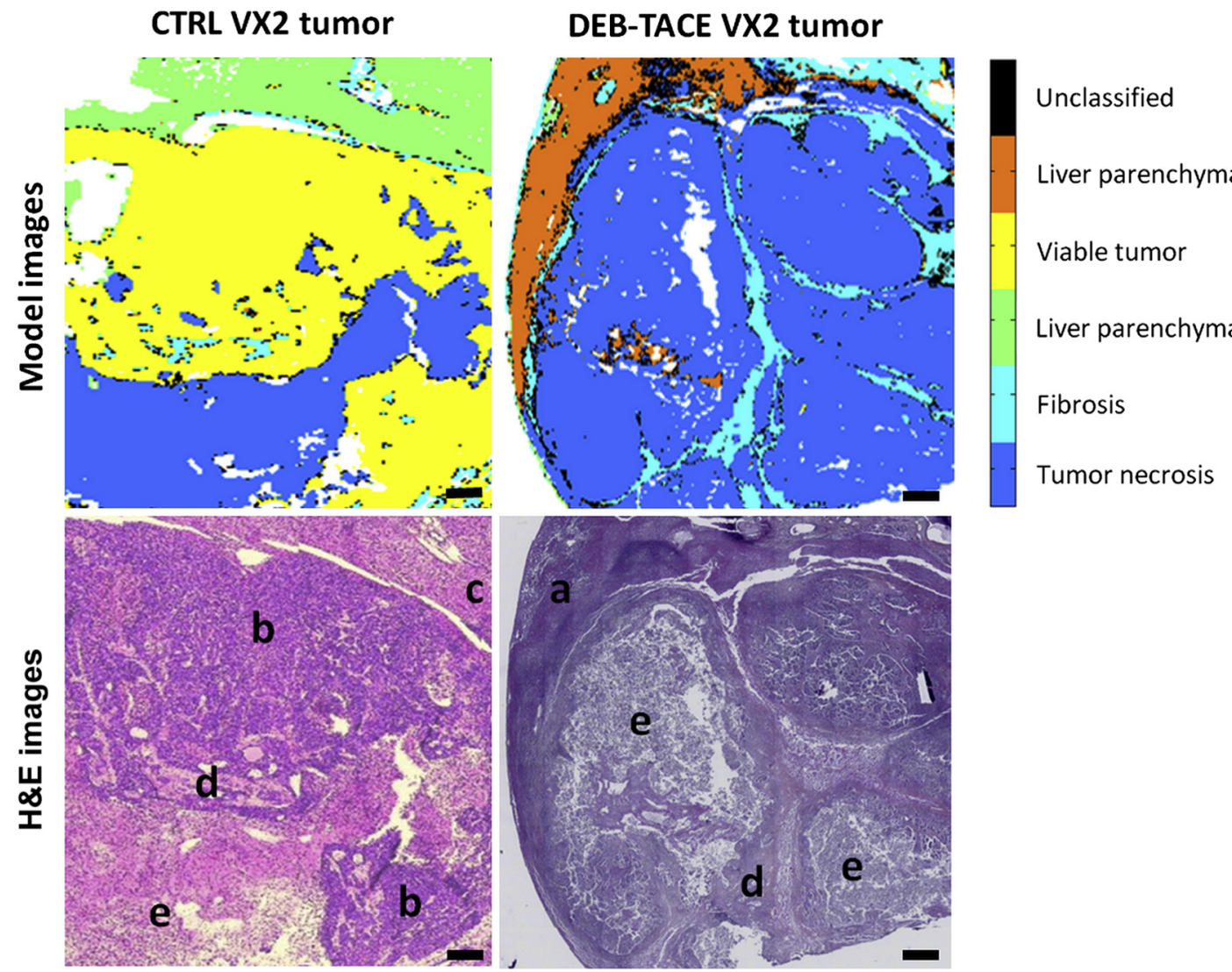

Figure 3 Linear discriminant analysis and hematoxylin-eosin-saffron-stained images of unknown VX2 tumor sections. Top: LDA images of VX2 tissue sections. Orange indicates liver parenchyma necrosis; yellow, viable tumor; dark blue, tumor necrosis; blue, fibrosis; green, liver parenchyma; and black, unclassified spectra. Bottom: Hematoxylin-eosin-saffron images of adjacent VX2 tissue sections.Scale bar $=1 \mathrm{~mm}$. a, liver parenchyma necrosis; $b$, viable tumor; $c$, liver parenchyma; CTRL, control; d, fibrosis; DEB-TACE, transarterial chemoembolization with drug-eluting beads; e, tumor necrosis.

Hamamatsu City, Japan). Measurements were performed with ICS FrameWork version 2.6 (Tribvn, Châtillon, France). We calculated correlations between the PCA-LDA model tissues quantification and histopathologic measurements using the Spearman coefficient $(r)$ for nonparametric correlation. $P<0.05$ was regarded as significant.

\section{VX2 Tissues Biochemical Analysis}

Because the spectral absorption bands are correlated to biochemical tissue composition, it is possible to determine which biomarkers varied according to the tissue type. The Matlab randfeatures function (available in the MatLab Statistics Toolbox and MatLab Bioinformatics Toolbox) was used to identify the most discriminant infrared wave numbers among our five populations of spectra (tumor necrosis, viable tumor, fibrosis, liver parenchyma, and liver parenchyma necrosis). ${ }^{37,38}$ The randfeatures function randomly selects a subset of 15 wave numbers and reduces all the spectra from 451 wave numbers to the 15 selected. The mean spectrum of each category was subtracted from the corresponding spectra. The resulting spectra were then classified using an LDA. After LDA classification, each spectrum is assigned percentages of belonging to each category. If the percentage of belonging to the correct category is over a confidence threshold fixed to $95 \%$, the percentages is considered well predicted. Then a performance threshold, fixed here to $95 \%$, is used to calculate the number of spectra correctly predicted. If this threshold is exceeded, the subset of 15 wave numbers is retained and a new iteration starts with 15 other wave numbers. Note that a wave number can be chosen several times. The randfeatures algorithm stops when 2000 subsets of 15 wave numbers are retained. The cardinality of each wave number, also called score, permits the determination of relevancy in the classification. The wave numbers are sorted according to their scores to determine the most discriminant ones.

\section{Results}

\section{Construction of the Predictive Model}

\section{KM Classification}

KM was applied to 14 infrared images from seven different VX2 tumors. KM clustering with two clusters number did not reveal evidence of any clear histologic structure (Figure 1A). Structures appeared for five cluster images. The areas corresponding to viable and necrotic tissues within the tumor were highlighted (Figure 1B). When comparing KM images 


\section{CTRL VX2 tumor}

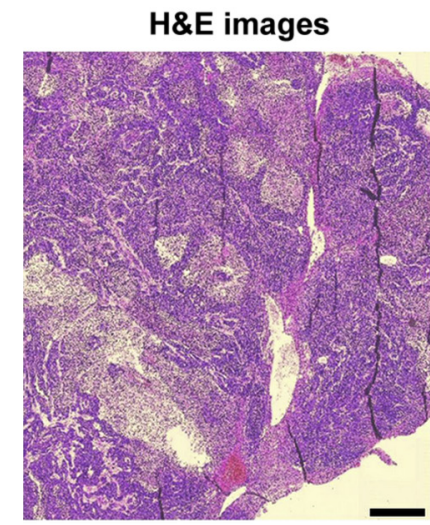

Tumor necrosis

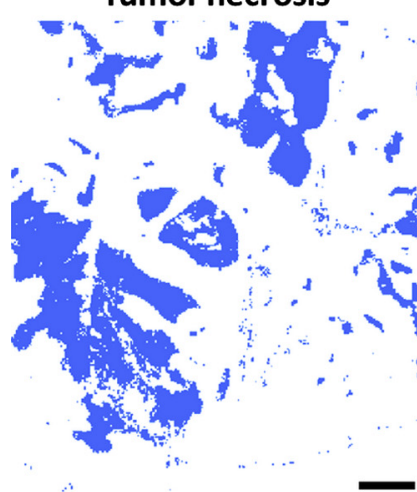

$25.1 \%$

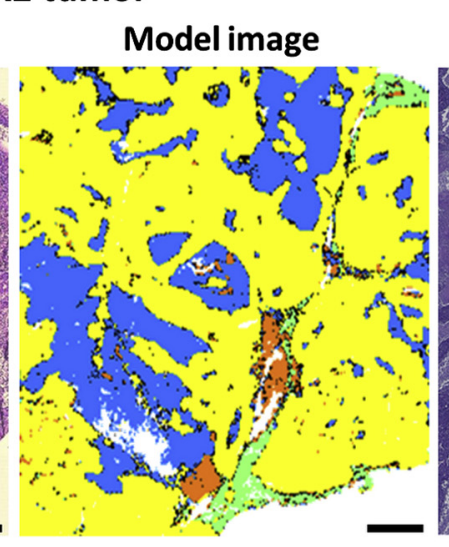

Viable tumor

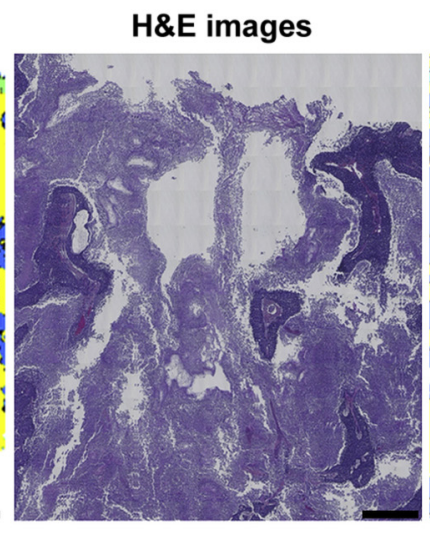

Tumor necrosis

$69.7 \%$

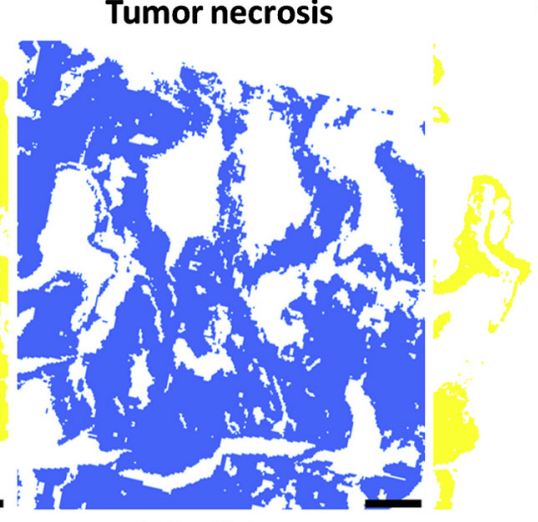

$88.6 \%$
DEB-TACE VX2 tumor

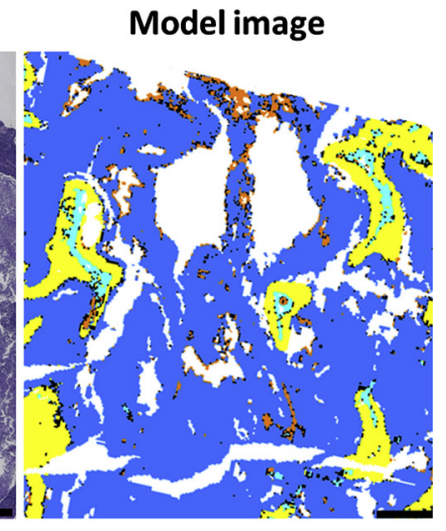

Viable tumor

Figure 4 Automatic tissue surface quantification by the principal component analysis and linear discriminant analysis model. Examples of tumor viability quantification on control (CTRL) VX2 tumor section and transarterial chemoembolization with drug-eluting beads (DEB-TACE)-treated VX2 tumor section. Surface is expressed as the percentage of pixels on images that are assigned to the viable tumor (yellow) and the necrotic tumor (dark blue) inside the tumor area. Scale bar $=1 \mathrm{~mm}$.

to HES staining, the sensitivity was higher than $93 \%$ for viable tumor, tumor necrosis, liver parenchyma, and liver parenchyma necrosis but was only $44 \%$ for fibrosis. With eight clusters, the correlation between KM images (Figure 1C) and HES staining (Figure 1D) was good for the five types of tissue. We observed $86.1 \%$ of matching areas for viable tumor (56/65; specificity, $95.5 \%), 94.6 \%$ for tumor necrosis $(71 / 75$; specificity, $94.5 \%), 80 \%$ for fibrosis $(36 / 45$; specificity, 96.9\%), $90 \%$ for liver parenchyma $(36 / 40$; specificity, $98.4 \%$ ), and $90 \%$ for liver parenchyma necrosis (27/30; specificity, 98.5\%). Increasing the number of clusters above eight did not increase the correspondence between KM images and HES-stained images and increased the number of unallocated areas (from $7.2 \%$ with eight clusters to $12.3 \%$ with 10 clusters). On the basis of the $\mathrm{KM}$ images in eight clusters, spectra assigned to histologic structures were included into a reference spectral database. The database contained 436,180 spectra.

\section{The PCA-LDA Model}

We calculated the PCA-LDA models with the training set by varying the number of principal components (two-thirds of spectra from the database or 290,786 spectra: 64,268 spectra for viable tumor, 124,463 for tumor necrosis, 32,259 for fibrosis, 33,621 for liver parenchyma, and 36,175 for liver parenchyma necrosis), and we tested them on the same validation set (one-third of spectra from the database or 145,394 spectra: 32,134 spectra for viable tumor, 62,232 for tumor necrosis, 16,129 for fibrosis, 16,811 for liver parenchyma, and 18,088 for liver parenchyma necrosis). The PCA-LDA model established with 20 PCs was chosen for the following study (Figure 2). It showed, for the five tissues of interest, sensitivity and specificity $>86.7 \%$ and $96.7 \%$ respectively.

\section{Application of the Predictive Model}

The LDA model was then applied to 24 spectral images collected from the 20 remaining VX2 tumors that were not included into the spectral database (Figure 3). The correlation between the 24 LDA images and HES staining was satisfactory for the five tissue types. We identified $92.9 \%$ (52/56; specificity, 96.8\%) of the areas sampled in normal liver parenchyma on HES-stained section that correctly matched with the green class assigned to liver parenchyma. In viable tumor, $89.4 \%$ (59/66; specificity, 96.3\%) of the areas selected on 
A
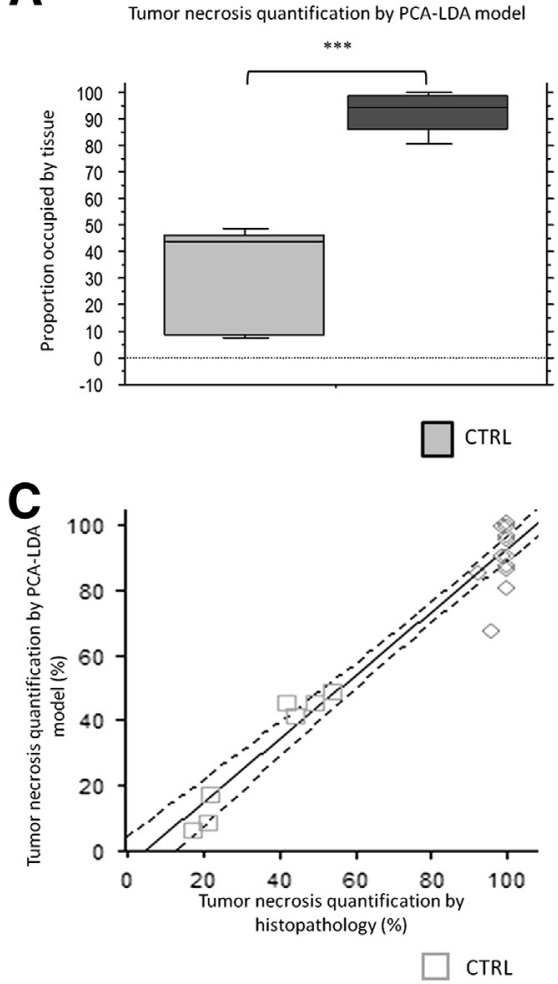

B
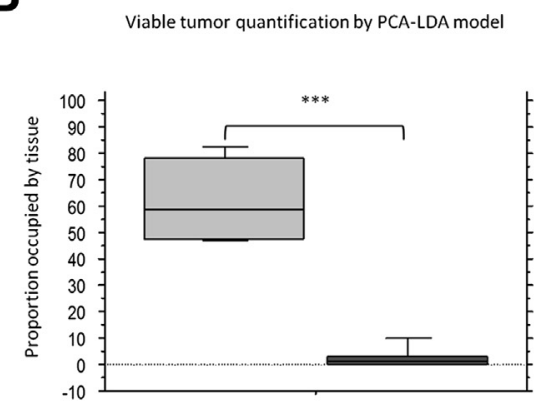

DEB-TACE

\section{D}

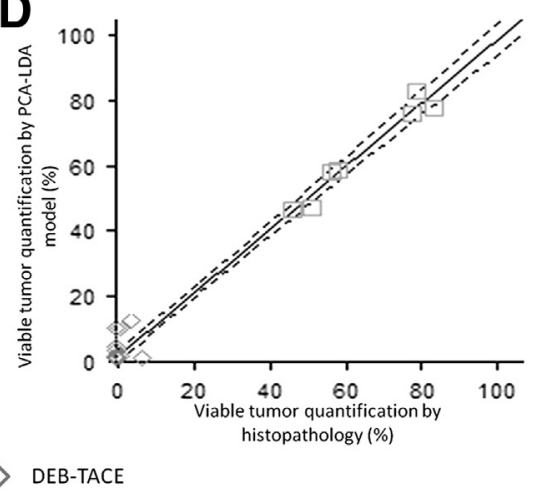

Figure 5 Proportion of necrotic and viable tumor evaluated by the principal component analysis (PCA) and linear discriminant analysis (LDA) model and correlation plots with histopathologic measurements. A: Tumor necrosis quantification by predictive model [control (CTRL): $33.1 \% \pm 19.6 \%$, median: $43.7 \%$; transarterial chemoembolization with drug-eluting beads (DEBTACE): $91.6 \% \pm 8.9 \%$; median, $94.6 \%$ ]. B: Viable tumor quantification by predictive model (CTRL: $62.2 \% \pm 15.2 \%$; median, $58.7 \%$; DEB-TACE: $2.6 \% \pm 4 \%$; median, $0.9 \%$ ). C: Correlation plots between values obtained with histopathologic analysis and predictive model for tumor necrosis tissue $\left(R^{2}=0.943\right)$. D: Correlation plots between values obtained with histopathologic analysis and predictive model for viable tumor tissue $\left(R^{2}=0.984\right)$. The linear regression is represented with the $95 \%$ CI for the mean (C and D). $* * * P<0.001$ (A and $\mathbf{B}$ ).
HES-stained section were present within the yellow class assigned to viable tumor, and in tumor necrosis, 94.8\% (73/77; specificity, $94.1 \%$ ) of the areas were in the dark blue class assigned to tumor necrosis. In fibrosis, $79.2 \%$ (42/53; specificity, $97.8 \%$ ) of the areas were assigned by the model to the blue class assigned to fibrosis. In liver parenchyma necrosis, $83.3 \%$ (40/48; specificity, $99.1 \%$ ) of the areas were assigned by the model to the orange class attributed to liver parenchyma necrosis.

The percentage of spectra assigned to each LDA class was automatically calculated by the model (Figure 4). For the DEB-TACE group, the LDA model determined that the surface of the necrotic tissue represented $91.6 \% \pm 9 \%$ of the tumor, whereas for the control group, it was $33.1 \% \pm 19.6 \%$ (Mann-Whitney $P=0.0004$ ) (Figure 5A). Concerning the viable tumor, the LDA model calculated $2.6 \% \pm 4 \%$ of the tumor for the DEB-TACE group and $62.2 \% \pm 15.2 \%$ for the control group (Mann-Whitney $P=0.0004$ ) (Figure 5B). The remaining percentages corresponded to fibrosis and unclassified spectra.

The histopathologic measurements revealed also a complete tumor necrosis in the DEB-TACE group (99.4\% $\pm 1.6 \%)$, whereas for the control group, it was $35.8 \% \pm 15.1 \%$ (MannWhitney $P<0.0001$ ). For the viable tumor, histopathologic measurements also revealed fewer viable tumors in the DEBTACE group $(0.57 \% \pm 1.6 \%)$ than in the control group $(64.2 \% \pm 15.1 \%)$ (Mann-Whitney $P<0.0001)$.

The correlation between both the techniques was calculated with the Spearman coefficient. We observed a significant correlation between the PCA-LDA model tissues quantification and histopathologic measurements for tumor necrosis $(r=0.827, P<0.0001)$ and viable tumor $(r=0.840, P<0.0001)$. The correlation plots between values obtained with histopathologic analysis and the PCALDA model give a $R^{2}$ of 0.943 for tumor necrosis (Figure 5C) tissue and a $R^{2}$ of 0.984 for viable tumor tissue (Figure 5D).

\section{VX2 Tissues Biochemical Analysis}

The statistical comparison between mean spectra of each tissue type allowed identifying the biochemical differences. The main spectral differences among the five tissue types of VX2 tumor were found in the infrared regions specific to protein (amide I at 1600 to $1700 \mathrm{~cm}^{-1}$, amide II at 1490 to $1575 \mathrm{~cm}^{-1}$, and amide III at to $\left.1350 \mathrm{~cm}^{-1}\right)$, glycogen $\left(1154,1080\right.$, and $\left.1025 \mathrm{~cm}^{-1}\right)$, sugar $\left(1000\right.$ to $\left.1200 \mathrm{~cm}^{-1}\right)$, and nucleic acid (1100 to $\left.1080 \mathrm{~cm}^{-1}\right)^{19}$ (Figure 6).

The application of the randfeatures function on two groups of spectra from two tissue types permitted to identify the biochemical changes involved in their discrimination. For example, between viable tumor and necrotic tumor tissues, we found a higher intensity of the infrared bands at $1538 \mathrm{~cm}^{-1}$ (amide II) and $1628 \mathrm{~cm}^{-1}$ (amide I) in necrotic tumor spectra. We also observed, on necrotic tumor spectra, a shift from 1658 to $1648 \mathrm{~cm}^{-1}$ of the amide I maximum intensity (Figure 7A). The differences between viable tumor and liver parenchyma tissues were due to the presence of strong absorption bands of glycogen (1154, 1080, and 1025 $\mathrm{cm}^{-1}$ ) in liver parenchyma spectra, whereas there was a 


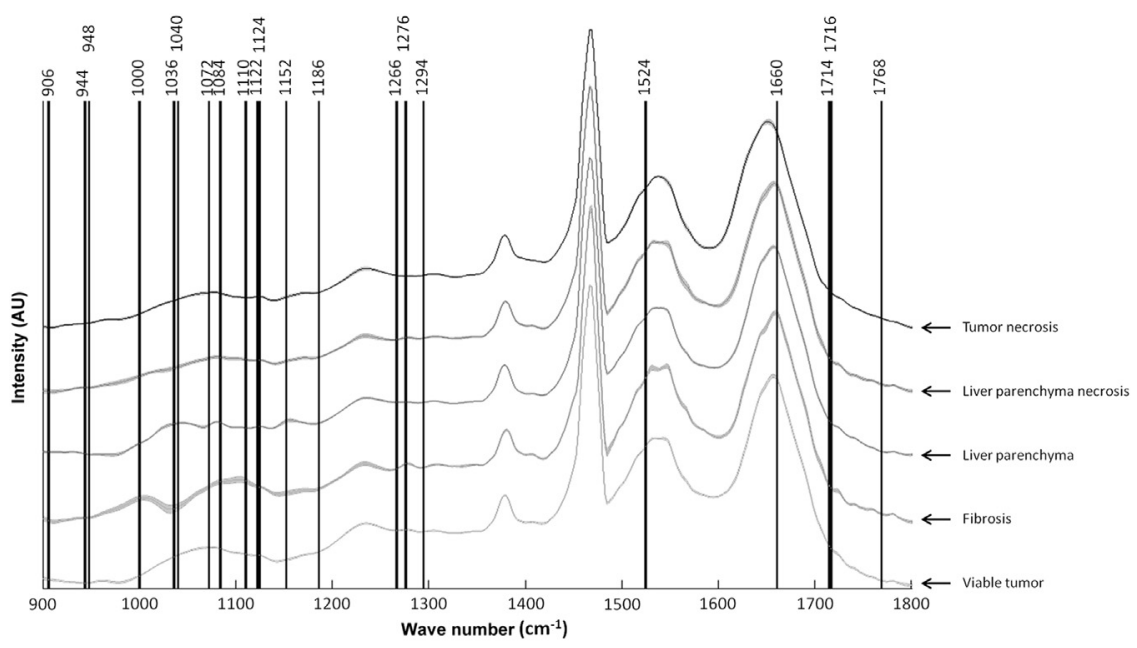

Figure 6 Main relevant infrared wave numbers to discriminate VX2 tumor tissues. Means \pm SD class spectra for viable tumor, fibrosis, liver parenchyma, liver parenchyma necrosis, and tumor necrosis. Offsets as marked were introduced for clarity. Vertical bars represent the most discriminant 21 wave numbers.

higher intensity of nucleic acids bands in viable tumor spectra $\left(1240,1080\right.$, and $968 \mathrm{~cm}^{-1}$ ) (Figure 7B).

\section{Discussion}

IRMS of tissue sections has been used to characterize a variety of neoplastic or disease states with the aim of helping pathologists in diagnosis. ${ }^{22,39}$ Our aims were to validate the use of infrared imaging to automatically quantify the VX2 tumor viability after DEB-TACE treatment, which is the recommended treatment for inoperable hepatocellular carcinoma. IRMS could provide a better understanding of posttherapy imaging appearance, which is essential for accurately assessing treatment response and choosing the best treatment option for patients.

\section{Construction of the Reliable and Predictive Model Based on References Samples}

The first step consisted of determining whether the main tissues composing the tumor and the host organ could be accurately differentiated with IRMS. Infrared images of unstained VX2 tissue sections were recorded, and spectral data from these acquisitions were analyzed with the KM classification method. The method is unsupervised, which means that the classification of the data is not determined by operator and solely depends on the similarities of the spectra. The comparison between the $\mathrm{KM}$ images processed in eight clusters and the HES-stained section suggests that the structures identified by the classification method correspond to the viable tumor, tumor necrosis, fibrosis, liver parenchyma, and liver parenchyma necrosis. To confirm this, we selected areas in each of the tissue types on the control HES and assessed which class they were assigned to on the infrared image. We obtained a correspondence $>80 \%$ with eight clusters classification, and increasing the number of clusters above eight did not improve this result. This method allowed the construction of a robust spectral database representative of each tissue type and was never performed by authors who simply used a visual comparison.

On the basis of KM images in eight clusters, spectra assigned to histologic structures were included in a reference spectral database. We included 436,180 spectra in our database, which represents a huge data set compared with other studies. We reduced the size of the data set with PCA. The PCA-LDA model calculated with 20 principal components revealed a good power of tissues identification (mean model sensitivity, 94.5\%). The 20 first principal components were sufficient to explain the variability of our data set, and to go beyond could constitute a risk of including variability due to noise. ${ }^{40}$ The PCA-LDA model revealed a sensitivity $>86.7 \%$ for the viable tumor, tumor necrosis, fibrosis, liver parenchyma, and liver parenchyma necrosis. The specificity was $>96.7 \%$ for the five types of tissue. These percentages are good compared with the values reported in studies of IRMS using the same type of predictive model. IRMS and predictive model recognition have been combined in a demonstration of this technique potential for automated histologic analysis of benign and malignant prostate tissues with sensitivity $>90 \% .{ }^{17}$ Ly et $\mathrm{al}^{29}$ measured a sensitivity varying between $74 \%$ and $98 \%$ in cutaneous carcinoma according to tissue type. Teh et al ${ }^{41}$ found a sensitivity of $95 \%$ and a specificity of $90 \%$ to identify dysplastic gastric tissue. Salman et $\mathrm{al}^{31}$ found that the normal and malignant cells from colonic tissue could be identified with approximately $86 \%$ of sensitivity. Nallala et $\mathrm{al}^{30}$ measured a sensitivity varying between $77 \%$ and 98\% in colon carcinoma according to tissue type.

\section{Application of the PCA-LDA Model on Test Samples and Tissue Quantification}

\section{External Validation of the PCA-LDA Model}

Although the use of an internal validation set is routinely performed to evaluate the performances of the predictive model, few studies have verified the accuracy of their model 

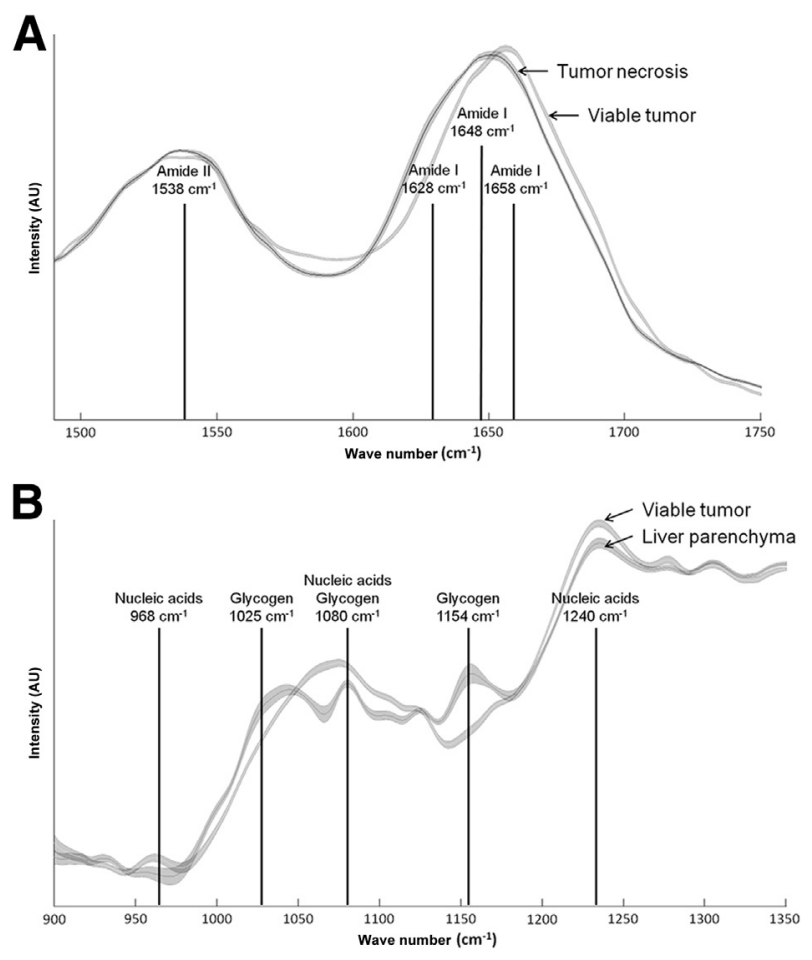

Figure 7 Main biochemical differences between viable tumor and necrotic tumor or liver parenchyma tissues. A: Means \pm SD class spectra for viable tumor and tumor necrosis. B: Means \pm SDs class spectra for viable tumor and liver parenchyma.

on a set of new test samples and compared the results with a reference technique. To our knowledge, this correlation step was never performed quantitatively on new infrared images but only on a new pool of infrared spectra. ${ }^{42-44}$ We only found two studies in which the authors applied their model to new tissue sections that have been used for neither model construction nor model internal validation..$^{29,30}$ The different structures of their samples could be identified on the infrared processed image, and the visual comparison to a control HES-stained section revealed good resemblance between the two images, but it was not assessed quantitatively.

We compared quantitatively the PCA-LDA images with adjacent HES-stained sections as described in Materials and Methods. We measured prediction accuracy from $79.2 \%$ to $94.8 \%$, depending on the tissues. These results reaffirm the potential of infrared imaging to automatically identify different tissue types only based on their infrared absorption spectra.

\section{Tissues Quantification}

\section{Effect of the DEB-TACE Treatment}

The automatic quantification of viable and necrotic tumor surface on the PCA-LDA images revealed a significant decrease of VX2 tumor viability after DEB-TACE treatment compared with the group of control tumors. After 3 days of treatment, the DEB induced a nearly complete necrosis $(91.6 \% \pm 8.9 \%)$ of the tumor with very few viable tumor areas $(2.6 \% \pm 4 \%)$. In the control tumors group, we observed a major proportion of viable tumor $(62.2 \% \pm 15.2 \%)$ with few necrotic areas $(33.1 \% \pm 19.6 \%)$ after 14 days of tumor development. Basically, during its rapid growth, the VX2 tumor necrotized spontaneously because of a flawed vasculature system. The necrosis corresponds to an ischemia necrosis.

Hong et $\mathrm{al}^{45}$ and Lee et $\mathrm{a}^{46}$ found that tumor necrosis in the DEB-TACE group reached $90 \%$ in the animals sacrificed 3 days after treatment and estimated that tumor necrosis did not exceed $35 \%$ after 14 days of development in the control group. Geschwind et $\mathrm{al}^{7}$ described that the mean fraction of viable tumor was approximately $55 \%$ of the whole tumor surface at 17 days after tumor implantation without treatment. Our findings were in a good accordance with later studies using the VX2 tumor model and further confirm the accuracy of IRMS to quantify objectively the antitumor effect after a DEB-TACE treatment.

\section{Correlation between IRMS and Histopathologic Methods}

Large areas of necrosis and small islands of necrotic areas were correctly identified by our predictive model. We observed a significant correlation between the PCA-LDA model tissues quantification and histopathologic measurements for viable tumor $(r=0.840, P<0.0001)$ and tumor necrosis $(r=0.827, P<0.0001)$. The infrared imaging technique is a good tool to characterize and quantify automatically the tissue types from unstained tissue sections. It is a complementary technique of histopathologic analysis that could be very helpful in quantifying precisely the viability of the VX2 tumor with a resolution close to cellular level. This study reveals a novel application of infrared imaging and provides a great solution to understand the tumor behavior under anticancer treatment.

\section{VX2 Tissues Biochemical Analysis}

Most spectral differences between liver parenchyma and viable tumor arise in the low-frequency region between 1200 and $900 \mathrm{~cm}^{-1}$ in which the glycogen and the skeletal vibrations of nucleic acids are observed. As previously reported, we observed an increase of nucleic acids absorption in cancerous tissues characterized by a broad band centered at $1080 \mathrm{~cm}^{-1}{ }^{47,48}$ This observation could be explained by the highest degree of cellular activity inside cancerous tissue, such as cells division and proliferation. ${ }^{48} \mathrm{We}$ detected several bands attributed to glycogen absorption $(1150,1080$, and $1028 \mathrm{~cm}^{-1}$ ) in liver parenchyma spectra that were not present in cancerous tissue spectra. The glycogen is stored in the liver by hepatocytes and could be metabolized in glucose during the glycogenolysis process. The absence of glycogen bands in the viable tumor tissue spectra indicated that tumor cells depleted their glycogen or they lost this storage function during malignancy. ${ }^{49}$

The differences between necrotic and viable tumor spectra result from a decrease in nucleic acid and an increase of protein signals that was also demonstrated by Beljebbar 
et al. ${ }^{50,51} \mathrm{We}$ also observed a shift of the amide I band from 1658 to $1648 \mathrm{~cm}^{-1}$ in the necrotic tumor spectra, which suggested a change in configuration of proteins. The higher intensity at $1628 \mathrm{~cm}^{-1}$ and the lower intensity at $1658 \mathrm{~cm}^{-1}$ in necrotic tumor spectra compared with viable tumor spectra revealed an increase of B-sheet structure and a decrease of $\alpha$-helix structure in the necrotic areas. ${ }^{52}$

\section{Limits}

Infrared images acquisition is still time-consuming (a mean of 3 hours per slide); however, the new generation of infrared microspectrometers is very promising. The coupling of focal plan array detectors to conventional FTIR systems and recent technical advances in focal plan array technology have allowed the concurrent rapid collection of thousands of infrared spectra over large areas of a sample. ${ }^{53}$

\section{Perspectives}

DEB-TACE revealed a good tumor response and an improved survival rate in patients who are not eligible for other treatments. ${ }^{54}$ Despite important advances in the field, DEB-TACE suffers from a lack of standardization in the selection of the type of DEB, the size of DEB, and the choice of chemotherapeutic agent. ${ }^{55}$ In this context, the next step will be to apply infrared imaging technique on VX2 tumors after different kind of DEB-TACE treatments. The automatic tissues characterization and quantification on these samples will help to assess the efficacy of these therapies and to determine the impact of each parameter. These processes may serve in the testing and implementation of novel combinatorial treatment strategies and in the determination of the best treatment option for patients.

Moreover, it is possible to detect the infrared signal of different molecules used in DEB treatment, such as doxorubicin $^{56,57}$ or ibuprofen. ${ }^{58}$ The intensity of the infrared signal obtained from a molecule is directly proportional to the concentration of this molecule as described by the Beer law. This mean could be used to quantify the drug concentration inside the DEB at different time points and determine the elution kinetic of the drug. ${ }^{57}$ IRMS could permit quantification on the same image the drug inside the beads, tissue damages, and biochemical changes induced by the treatment. ${ }^{19,59}$

Another perspective would be to use IRMS to characterize other tissues of interest, such as inflammatory tissue or vascular tissue, which have a specific infrared spectral signature. ${ }^{60-62}$ It would be also interesting to investigate whether IRMS can discriminate between the spontaneous necrosis of VX2 tumors and the necrosis induced by anticancerous treatments, which is not possible by HES staining.

\section{Conclusion}

IRMS coupled with predictive model analysis allows the automatic identification and quantification of the surface of the main tissue types on histologic sections of rabbit VX2 liver tumor before and after DEB-TACE treatment. IRMS is able to provide, on the same image, biochemical and morphologic information that is essential for accurately assessing treatment response and understanding posttherapy imaging appearance. IRMS could play an important role in the preclinical field, where current imaging tools have bad resolution (positron emission tomography is sensitive but works at the millimeter scale) and/or poor sensitivity (magnetic resonance imaging needs contrast agents to highlight changes in molecular concentrations). The ideal technique does not exist, but all techniques have distinct advantages, and their association could provide a great overview of tumor behaviors under anticancer treatments.

\section{Acknowledgments}

We thank Cyril Gobinet for analytical help and Saida Homayra for histopathologic examination. We also thank the Tissular and Cellular Imaging Platform (Reims University).

\section{References}

1. Kidd JG, Rous P: A transplantable rabbit carcinoma originating in a virus-induced papilloma and containing the virus in masked or altered form. J Exp Med 1940, 71:813-838

2. Rous P, Kidd JG, Smith WE: Experiments on the cause of the rabbit carcinomas derived from virus-induced papillomas. II. Loss by the Vx2 carcinoma of the power to immunize hosts against the papilloma virus. J Exp Med 1952, 96:159-174

3. Tu M, Xu L, Wei X, Miao Y: How to establish a solitary and localized VX2 lung cancer rabbit model? a simple and effective intrapulmonary tumor implantation technique. J Surg Res 2009, 154: 284-292

4. Ke S, Ding XM, Kong J, Gao J, Wang SH, Cheng Y, Sun WB: Low temperature of radiofrequency ablation at the target sites can facilitate rapid progression of residual hepatic VX2 carcinoma. J Trans1 Med 2010, 8:73

5. Liang XM, Tang GY, Cheng YS, Zhou B: Evaluation of a rabbit rectal VX2 carcinoma model using computed tomography and magnetic resonance imaging. World J Gastroenterol 2009, 15:2139-2144

6. Ramirez LH, Juliéron M, Bonnay M, Koscielny S, Zhao Z, Gouyette A, Munck JN: Stimulation of tumor growth in vitro and in vivo by suramin on the VX2 model. Invest New Drugs 1995, 13:51-53

7. Geschwind JF, Artemov D, Abraham S, Omdal D, Huncharek MS, McGee C, Arepally A, Lambert D, Venbrux AC, Lund GB: Chemoembolization of liver tumor in a rabbit model: assessment of tumor cell death with diffusion-weighted MR imaging and histologic analysis. J Vasc Interv Radiol 2000, 11:1245-1255

8. Zheng LF, Li YJ, Wang H, Zhao JL, Wang XF, Hu YS, Zhang GX: Combination of vascular endothelial growth factor antisense oligonucleotide therapy and radiotherapy increases the curative effects against maxillofacial VX2 tumors in rabbits. Eur J Radiol 2011, 78: $272-276$

9. Han K, Wang Z, Peng X, Chen B, Wen X, Dong Y, Wu C: Transarterial chemoembolization using docetaxel-loaded phytantriol cubic phase precursor for the treatment of hepatocellular carcinoma. J Pharm Sci 2010, 100:2240-2247

10. Kang J, Wu X, Wang Z, Ran H, Xu C, Wu J, Wang Z, Zhang Y: Antitumor effect of docetaxel-loaded lipid microbubbles combined with ultrasound-targeted microbubble activation on VX2 rabbit liver tumors. J Ultrasound Med 2010, 29:61-70 
11. Nitta N, Sonoda A, Seko A, Ohta S, Nagatani Y, Tsuchiya K, Otani H, Tanaka T, Kanasaki S, Takahashi M, Murata K: A combination of cisplatin-eluting gelatin microspheres and flavopiridol enhances anti-tumour effects in a rabbit VX2 liver tumour model. Br J Radiol 2010, 83:428-432

12. Bruix J, Sherman M, Llovet JM, Beaugrand M, Lencioni R, Burroughs AK, Christensen E, Pagliaro L, Colombo M, Rodes J: Clinical management of hepatocellular carcinoma: conclusions of the Barcelona-2000 EASL conference. J Hepatol 2001, 35:421-430

13. Livraghi T, Goldberg SN, Lazzaroni S, Meloni F, Ierace T, Solbiati L, Gazelle GS: Hepatocellular carcinoma: radio-frequency ablation of medium and large lesions. Radiology 2000, 214:761-768

14. Yan K, Chen MH, Yang W, Wang YB, Gao W, Hao CY, Xing BC, Huang XF: Radiofrequency ablation of hepatocellular carcinoma: long-term outcome and prognostic factors. Eur J Radiol 2008, 67: 336-347

15. Cook ES, Jansen CH, Kreke CW, Motzel W: A new tool for cancer investigation: qualitative and quantitative infrared spectroscopy of proteins and enzymes. Acta Unio Int Contra Cancrum 1956, 12:503-507

16. Fong CT, Lippincott SW, Eriksen N: Infrared spectroscopy of crystalline albumin in human neoplasia. J Natl Cancer Inst 1957, 18: $271-275$

17. Fernandez DC, Bhargava R, Hewitt SM, Levin IW: Infrared spectroscopic imaging for histopathologic recognition. Nat Biotechnol 2005, 23:469-474

18. Diem M, Papamarkakis K, Schubert J, Bird B, Romeo MJ, Miljković M: The infrared spectral signatures of disease: extracting the distinguishing spectral features between normal and diseased states. Appl Spectrosc 2009, 63:307A-318A

19. Bellisola G, Sorio C: Infrared spectroscopy and microscopy in cancer research and diagnosis. Am J Cancer Res 2012, 2:1-21

20. Wang JS, Shi JS, Xu YZ, Duan XY, Zhang L, Wang J, Yang LM, Weng SF, Wu JG: FT-IR spectroscopic analysis of normal and cancerous tissues of esophagus. World J Gastroenterol 2003, 9: 1897-1899

21. Beleites C, Steiner G, Sowa M, Baumgartner R, Sobottka S, Schackert G, Salzer R: Classification of human gliomas by infrared imaging spectroscopy and chemometric image processing. Vibrational Spectroscopy 2005, 38:143-149

22. Kendall C, Isabelle M, Bazant-Hegemark F, Hutchings J, Orr L, Babrah J, Baker R, Stone N: Vibrational spectroscopy: a clinical tool for cancer diagnostics. Analyst 2009, 134:1029-1045

23. Meurens M, Wallon J, Tong J, Noel H, Haot J: Breast cancer detection by Fourier transform infrared spectrometry. Vibrational Spectroscopy 1996, 10:341-346

24. Lasch P, Haensch W, Naumann D, Diem M: Imaging of colorectal adenocarcinoma using FT-IR microspectroscopy and cluster analysis. Biochim Biophys Acta 2004, 1688:176-186

25. Dukor RK: Vibrational spectroscopy in the detection of cancer. Handbook of vibrational spectroscopy. Hoboken, NJ, Wiley, 2002, $3335-3361$

26. Gaigneaux A, Ruysschaert JM, Goormaghtigh E: Infrared spectroscopy as a tool for discrimination between sensitive and multiresistant K562 cells. Eur J Biochem 2002, 269:1968-1973

27. Gazi E, Dwyer J, Lockyer N, Gardner P, Vickerman JC, Miyan J, Hart CA, Brown M, Shanks JH, Clarke N: The combined application of FTIR microspectroscopy and ToF-SIMS imaging in the study of prostate cancer. Faraday Discuss 2004, 126:41-59. discussion 77-92

28. Krafft C, Sobottka SB, Geiger KD, Schackert G, Salzer R: Classification of malignant gliomas by infrared spectroscopic imaging and linear discriminant analysis. Anal Bioanal Chem 2007, 387:1669-1677

29. Ly E, Piot O, Durlach A, Bernard P, Manfait M: Differential diagnosis of cutaneous carcinomas by infrared spectral micro-imaging combined with pattern recognition. Analyst 2009, 134:1208-1214

30. Nallala J, Diebold MD, Gobinet C, Bouché O, Sockalingum GD, Piot O, Manfait M: Infrared spectral histopathology for cancer diagnosis: a novel approach for automated pattern recognition of colon adenocarcinoma. Analyst 2014, 139:4005-4015

31. Salman A, Argov S, Ramesh J, Goldstein J, Sinelnikov I, Guterman H, Mordechai S: FT-IR microscopic characterization of normal and malignant human colonic tissues. Cell Mol Biol 2001, 47: OL159-166

32. Ly E, Piot O, Wolthuis R, Durlach A, Bernard P, Manfait M: Combination of FTIR spectral imaging and chemometrics for tumour detection from paraffin-embedded biopsies. Analyst 2008, 133: 197-205

33. Wolthuis R, Travo A, Nicolet C, Neuville A, Gaub MP, Guenot D, Ly E, Manfait M, Jeannesson P, Piot O: IR spectral imaging for histopathological characterization of xenografted human colon carcinomas. Anal Chem 2008, 80:8461-8469

34. Kohler A, Kirschner C, Oust A, Martens H: Extended multiplicative signal correction as a tool for separation and characterization of physical and chemical information in Fourier transform infrared microscopy images of cryo-sections of beef loin. Appl Spectrosc 2005, $59: 707-716$

35. Martens H, Stark E: Extended multiplicative signal correction and spectral interference subtraction: new preprocessing methods for near infrared spectroscopy. J Pharm Biomed Anal 1991, 9:625-635

36. Travo A, Piot O, Wolthuis R, Gobinet C, Manfait M, Bara J, ForgueLafitte ME, Jeannesson P: IR spectral imaging of secreted mucus: a promising new tool for the histopathological recognition of human colonic adenocarcinomas. Histopathology 2010, 56:921-931

37. Nguyen TT, Happillon T, Feru J, Brassartâ Passco S, Angiboust JFo, Manfait M, Piot O: Raman comparison of skin dermis of different ages: focus on spectral markers of collagen hydration. J Raman Spectrosc 2013, 44:1230-1237

38. Poplineau M, Trussardi-Régnier Al, Happillon T, Dufer J, Manfait M, Bernard P, Piot O, Antonicelli F: Raman microspectroscopy detects epigenetic modifications in living Jurkat leukemic cells. Epigenomics 2011, 3:785-794

39. Krafft C, Steiner G, Beleites C, Salzer R: Disease recognition by infrared and Raman spectroscopy. J Biophotonics 2009, 2:13-28

40. Cangelosi R, Goriely A: Component retention in principal component analysis with application to cDNA microarray data. Biol Direct 2007, 2:1-21

41. Teh S, Zheng W, Ho K, Teh M, Yeoh K, Huang Z: Diagnostic potential of near-infrared Raman spectroscopy in the stomach: differentiating dysplasia from normal tissue. Br J Cancer 2008, 98: $457-465$

42. Bergner N, Romeike BF, Reichart R, Kalff R, Krafft C, Popp J: Tumor margin identification and prediction of the primary tumor from brain metastases using FTIR imaging and support vector machines. Analyst 2013, 138:3983-3990

43. Gazi E, Dwyer J, Gardner P, Ghanbari-Siahkali A, Wade AP, Miyan J, Lockyer NP, Vickerman JC, Clarke NW, Shanks JH, Scott LJ, Hart CA, Brown M: Applications of Fourier transform infrared microspectroscopy in studies of benign prostate and prostate cancer: a pilot study. J Pathol 2003, 201:99-108

44. Lecellier A, Mounier J, Gaydou V, Castrec L, Barbier G, Ablain W, Manfait M, Toubas D, Sockalingum GD: Differentiation and identification of filamentous fungi by high-throughput FTIR spectroscopic analysis of mycelia. Int J Food Microbiol 2014, 168-169:32-41

45. Hong K, Khwaja A, Liapi E, Torbenson MS, Georgiades CS, Geschwind JF: New intra-arterial drug delivery system for the treatment of liver cancer: preclinical assessment in a rabbit model of liver cancer. Clin Cancer Res 2006, 12:2563-2567

46. Lee K-H, Liapi EA, Cornell C, Reb P, Buijs M, Vossen JA, Ventura VP, Geschwind JFH: Doxorubicin-loaded QuadraSphere microspheres: plasma pharmacokinetics and intratumoral drug concentration in an animal model of liver cancer. Cardiovasc Intervent Radiol 2010, 33:576-582

47. Benedetti E, Bramanti E, Papineschi F, Rossi I, Benedetti E: Determination of the relative amount of nucleic acids and proteins in 
leukemic and normal lymphocytes by means of Fourier transform infrared microspectroscopy. Appl Spectrosc 1997, 51:792-797

48. Chiriboga L, Yee H, Diem M: Infrared spectroscopy of human cells and tissue, part VI: a comparative study of histopathology and infrared microspectroscopy of normal, cirrhotic, and cancerous liver tissue. Appl Spectrosc 2000, 54:1-8

49. Mehrotra R, Gupta A, Kaushik A, Prakash N, Kandpal H: Infrared spectroscopic analysis of tumor pathology. Indian J Exp Biol 2007, 45:71

50. Beljebbar A, Amharref N, Leveques A, Dukic S, Venteo L, Schneider L, Pluot M, Manfait M: Modeling and quantifying biochemical changes in C6 tumor gliomas by Fourier transform infrared imaging. Anal Chem 2008, 80:8406-8415

51. Beljebbar A, Dukic S, Amharref N, Manfait M: Screening of biochemical/histological changes associated to C6 glioma tumor development by FTIR/PCA imaging. Analyst 2010, 135:1090-1097

52. Yamada T, Miyoshi N, Ogawa T, Akao K, Fukuda M, Ogasawara T, Kitagawa Y, Sano K: Observation of molecular changes of a necrotic tissue from a murine carcinoma by Fourier-transform infrared microspectroscopy. Clin Cancer Res 2002, 8:2010-2014

53. Dorling KM, Baker MJ: Rapid FTIR chemical imaging: highlighting FPA detectors. Trends Biotechnol 2013, 31:437-438

54. Muros-Ortega M, Diaz-Carrasco MS, Capel A, Calleja MA, Martinez F: Effectiveness and safety of doxorubicin loaded beads in hepatocellular carcinoma. Int J Clin Pharm 2013, 35:1105-1112

55. Marelli L, Stigliano R, Triantos C, Senzolo M, Cholongitas E, Davies N, Tibballs J, Meyer T, Patch DW, Burroughs AK:
Transarterial therapy for hepatocellular carcinoma: which technique is more effective? a systematic review of cohort and randomized studies. Cardiovasc Intervent Radiol 2007, 30:6-25

56. Namur J, Citron SJ, Sellers MT, Dupuis MH, Wassef M, Manfait M, Laurent A: Embolization of hepatocellular carcinoma with drugeluting beads: doxorubicin tissue concentration and distribution in patient liver explants. J Hepatol 2011, 55:1332-1338

57. Namur J, Wassef M, Millot JM, Lewis AL, Manfait M, Laurent A: Drug-eluting beads for liver embolization: concentration of doxorubicin in tissue and in beads in a pig model. J Vasc Interv Radiol 2010, 21:259-267

58. Namur J, Wassef M, Pelage JP, Lewis A, Manfait M, Laurent A: Infrared microspectroscopy analysis of ibuprofen release from drug eluting beads in uterine tissue. J Control Release 2009, 135:198-202

59. Derenne A, Verdonck M, Goormaghtigh E: The effect of anticancer drugs on seven cell lines monitored by FTIR spectroscopy. Analyst 2012, 137:3255-3264

60. Diem M, Chiriboga L, Yee H: Infrared spectroscopy of human cells and tissue, VIII: strategies for analysis of infrared tissue mapping data and applications to liver tissue. Biopolymers 2000, 57:282-290

61. Li QB, Sun XJ, Xu YZ, Yang LM, Zhang YF, Weng SF, Shi JS, Wu JG: Use of Fourier-transform infrared spectroscopy to rapidly diagnose gastric endoscopic biopsies. World J Gastroenterol 2005, 11:3842-3845

62. Wehbe K, Pineau R, Eimer S, Vital A, Loiseau H, Deleris G: Differentiation between normal and tumor vasculature of animal and human glioma by FTIR imaging. Analyst 2010, 135:3052-3059 